R. Bubeck

S. Neser

C. Bechinger

P. Leideret

\title{
Structure and dynamics of two-dimensional colloidal crystals in confined geometry
}

\author{
Abstract The properties of two- \\ dimensional (2D) colloidal crystals \\ have been widely investigated during \\ the Jast 20 years, but only little \\ attention has been paid to effects of \\ finite system size. We use video- \\ microscopy to study small $2 \mathrm{D}$ crystals \\ consisting of approximately 10 to 100 \\ particles confined in hexagonal, \\ circular and square regions. The \\ observed crystal structures are
}

strongly dominated by the shape of those regions and anisotropic diffusion coefficients, indicating a reduced mobility perpendicular to the walls.

\section{Key words Two-dimensional} crystallization - colloidal crystals confinement - restricted geometry finite-size effects
The fascinating melting and freezing properties of two dimensional (2D) systems motivated a lot of scientific work during the past years [1-3]. In 1973 Kosterlitz. and Thouless proposed a two step melting scenario where melting is mediated by the dissociation of paired lattice faults [1]. In the following years this theory was refined by Halperin, Nelson and Young [2]. In contrast to the 3D case where both translational and bond orientational order are lost at the melting temperature $T_{m}$, the KTHNY theory predicts a new intermediate hexatic phase where translational order is lost but, some bond orientational order is still present. Experimental data are available for many different 2D systems, like electrons on helium, noble gases adsorbed on graphite and colloidal spheres confined between two walls or at an interface [3]. The great advantage of colloidal systems are their convenient time (milliseconds) and length scales (microns) which allow the observation of single particle trajectories and the variety of available interaction potentials.

In this paper we present experimental data on the structure and dynamics of laterally confined 2D colJoidal systems consisting of large superparamagnetic spheres lying on a smooth polymer substrate. A similar system has been already used by Zahn et al. [4] for the investigation of melting in large 2D systems. The lateral confinement is obtained by patterning the surface. An external magnetic field $\mathbf{B}$ perpendicular to the $2 \mathrm{D}$ plane is used to generate a magnetic moment $\mathbf{M}$ within the particles leading to a repulsive pair potential of the form $\mathrm{V} \propto M^{2} / r^{3}$, where $r$ is the distance between two particles. In this system the plasma parameter $\Gamma$, defined as ratio between the magnetic energy $U$ and $k_{\mathrm{B}} T$, depends on both the external magnetic field $B$ and the temperature $T$. For reasons of convenience, in this system usually temperature is kept constant and phase transitions are observed as a function of the magnetic field $B$. It is noticeable, that the well known interaction allowed a comparison of the experiment and theory without any free parameter! [4].

The sample cell (Fig. 1a) is formed by (wo circular fused silica plates which are fixed at a distance of $1 \mathrm{~mm}$ by an $\mathrm{O}$-ring. To reduce the sticking of the particles, a smooth 3-4 $\mu \mathrm{m}$ thick film of poly(methyl-methacrylate) (PMMA) was spio coated on top of the bottom silica plate. To realize the lateral confinement we then applied thin structured copper foils onto the PMMA substrate. These foils which are commercially available in a variety of sizes and geometries as grids for Transmission Electron Microscopy (TEM), consist of a large number $(20-100)$ of identical 


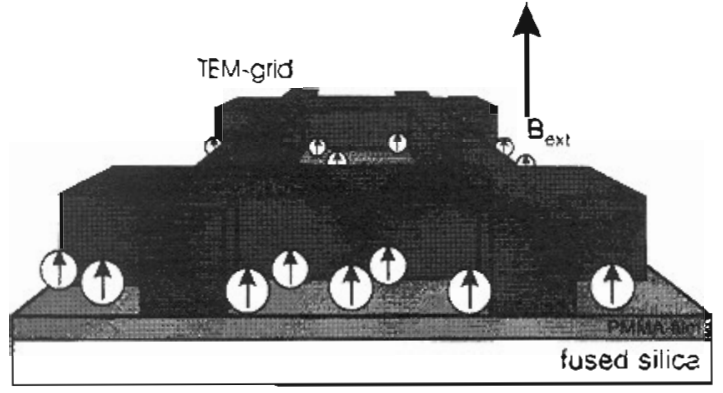

(a)

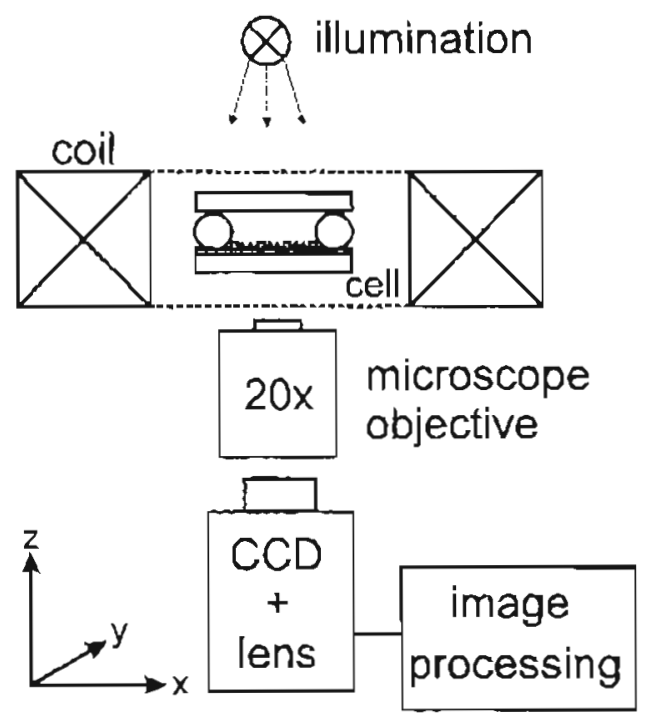

(b)

Fig. 1 Experimental setup. (a) Magnification of the patterned silica plate at the bottom of the cell: paramagnetic colloidal particles with induced magretic moments are lying on a PMMA film. A TEM grid serves as lateral confinement for the particles. (b) lnverted microscope consisting of NPLAN $20 \times 0.4$ objective, CCD-camera with $f=135 \mathrm{~mm}$ lens and Köhlerian illumination unit. A coil is used to generate a magnetic field inside the cell

holes with very narrow mechanical tolerances separated by small bridges. The large number of identical structures greatly improves statistics. To fix a grid we heated it up to a temperature of $150^{\circ} \mathrm{C}$ and pressed it onto the PMMAfilm. By this process the grid is then glued onto the PMMA film which is only slightly distorted by this procedure. Consequently, the walls of the compartments are formed by the bridges of the grid, whereas the bottom of the confined areas consists of PMMA.

After insertion of the colloidal suspension (DynaBeads $4.5 \mu \mathrm{m}$, Fa. Dynal, Lot-No. A44610, $\Phi_{0} \approx 1 \times 10^{-4}$ ) which was additionally stabilized with 0.2 wt $\%$ Triton $X-100$, gravity causes the particles to sediment onto the bottom plate. Parlicles which sediment onto the bridges perform a Brownian random walk and therefore are also trapped in the holes within a few minutes. This results in the forma- tion of a 2D colloidal system whose particle density can be tuned via the initial volume fraction $\Phi_{0}$. Since the displacement of a particle a tenth of its diameter against gravity would increasc its potential energy by about $18 k_{B} T$ the assumption for the system to be 2D is well fulfilled.

Our experimental setup is shown in Fig. $1 \mathrm{~b}$. The cell is placed in the center axis of a copper coil which can generate magnetic ficlds of up to $12 \mathrm{mT}$. The particles were imaged with an inverted microscope which consists of a Leica NPLAN $20 \times 0.4$ objective, a CCD camera equipped with a $f=135 \mathrm{~mm}$ lens and a Köhlerian illumination unit. This setup provides a homogeneously illuminated field of view with $550 \times 450$ microns in size. Data are taken with an image processing system connected to a computer which allows to calculate particle trajectories $\mathbf{r}(t)=(x(t)$, $y(t))$ with a spatial resolution of $0.5 \mu \mathrm{m}$ and a temporal resolution of $20 \mathrm{~ms}$. In the absence of an external magnetic field the particles behave like hard spheres. To characterize the dynamical behaviour of the system we consider the mean square displacement

$\Delta r^{2}(\tau)=\left\langle\left(\Gamma_{i}(t+\tau)-r_{i}(t)\right)^{2}\right\rangle_{t, i}$

where $\tau$ denotes the considered time interval and $r_{i}(t)$ is the position vector of the ith particle at the time $t$, which - in the absence of external forces - shows a linear increase in time and can be described by the usual 2D diffusion law

$\Delta r^{2}(\tau)=4 D \tau$,

where $D$ denotes the diffusion constant. If external lateral forces are present nonlinear terms may appear: e.g. when the 2D plane is not exactly horizontal, gravity may superimpose a term $\propto \tau^{2}$ in $\Delta r^{2}(\tau)$ (corresponding to a constant drift velocity). To avoid this, before each measurement the apparatus is calibrated until the square component of a fit to the measured $\Delta r^{2}(\tau)$ is negligible within the resolution of the apparatus.

For a system where no boundaries are present, i.e. no TEM grid is glued onto the PMMA film, we find a diffusion constant $D=0.035(1) \mu \mathrm{m}^{-2} \mathrm{~s}^{-1}$ parallel to the surface corresponding to about $36 \%$ of the free diffusion constant $D_{0}$ as calculated with the Stokes-Einstein equation (see Fig. 2). This result is consistent with the picture drawn by Faucheux et al. [5] who predicted and measured a reduction of the free diffusion up to a third of $D_{0}$ in the case of colloidal particles moving on a flat surface.

When applying a magnetic field the behaviour of the mean square displacement changes significantly. In Fig. 2 we plotted the mean square displacements as a function of time for different magnetic fields. For large values of $\tau \Delta r^{2}(\tau)$ still behaves linearly with a slope which decreases with increasing magnetic field strength. This dependence of $D_{\text {long }}^{\text {self }}$ on $B$ is also shown in the inset of Fig. 2, where one can see that the decrease is strongest for small fields while 


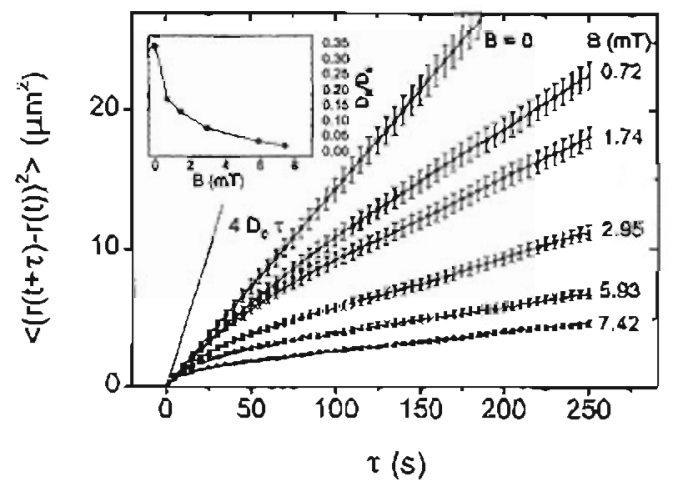

Fig. 2 Mcan square displacements of $\approx 600$ particles which are part of a infinite systero: Increasing the magnetic field reduces the longtime self-diffusion and increases the localization of the parlicles. In the inset the long tine self diffusion coefficient $D_{l o n g}^{\text {self }}$ is plotted over the applico magnetic ficld $B$

$\Delta r^{2}(\tau)$ approaches a constant for large values of $B$. For small $\tau$ 's there is a cross-over regime where the slope of $\Delta r^{2}(\tau)$ approximately increases to the value found when no magnetic field was present. This experimental observation is consistent with the well known crossover from shorttime 10 long time self diflusion: On sbort time scales a particle can diffuse (reely $\left(D=D_{0}^{\text {solf }}\right)$ in the cage formed by its nearest neighbours. With increasing time $\Delta r^{2}(\tau)$ cannot increase linearly anymore because the particle starts to sample the energy well of the surrounding spheres and a deformation of the cage would be neccessary for a further movement in the same direction $\left(D=D_{\text {long }}^{\text {velf }}\right)$. With increasing field strength the interaction becomes stronger and thus $D_{\text {loog }}^{\text {self }}$ decreases until finally - in the solid phase it becomes extremely unlikely that a particle leaves its cage. Therefore the mean square displacement becomes a constant and $D_{\text {long }}^{\text {scli }}$ approaches zero. ${ }^{1}$ A qualitatively similar behaviour has been found by Zahn ef al. [4] investigating the same colloidal particles at the water air interface within a small water droplet. Alter this brief discussion of the behaviour of the extended system, the effects of finite system sizes will be considered: In contrast 10 infinite $2 \mathrm{D}$ systems where the hexagonal lattice is the cnergetically preferred structure, in finite systems tbe equjlibrium structure for a given number of particles strongly depends on the size and geometry of the confinement. To get these equilibriucn structures we slowly increased the external magneric field up to values of $12 \mathrm{mT}$ with a rate of approximately $1 \mathrm{mT}$ per hour. This small rate is needed because of the slow dynamics for our large spheres, i.e. the diffusion constant without applied magnetic field of $D=0.035 \mu \mathrm{m} / \mathrm{s}$ corresponds to a time of $150 \mathrm{~s}$ for a particle to diffuse a distance of its own diameter. This fact leads to long siructural relaxation times of several hours. In the following we show the crystal structures in the case of high magnetic field's for different confinement geometries.

\section{Hexagonal symmotry}

An example for hexagonal confinement is shown in Fig. 3a. The orientation of the whole lattice is aligned to that of the confinement region and one clearly observes the hexagonal symmetry. Consequently, the particles are arranged in hexagonal "shells" around the center particle. For infinite 2D systems the hexagonal lattice is distinguished as the equilibrium structure of the crystalline phase [6]. Therefore, one would expect that confining particles to a hexagonal region should lead to the formation of a triangular lattice. However, this only holds for some magic numbers where the colloidal crystat and the confinement region are commensurate. One can easily calculate that if $S$ denotes the number of hexagonal shells, a crystal of $1+3 S(S+1)$ particles fies exactly into a bexagonal shape, otherwise distortions will occur [7]. In the example shown in Fig. 3a, there are 4 shells with a total number of 59 particles. However, since a perlect crystal requires 61 instead of 59 particles, slight distortions in the fourth shell are found.

\section{Square symmetry}

In this case, due to the competition of the intriasic hexagonal symmetry and the extrinsic square symmetry the dependence of structure formation on the particle number $N$ is even more pronounced. This can be demonstrated easily when comparing two systems of consecutive particle numbers (Fig. 4a and b). In Fig. 4a is considered a system of 16 particles in a square of $90 \mu \mathrm{m}$ sidelength. The particles are found to arrange themselves in a $4 \times 4$ square lattioe, i.e. the structure is only determined by the square shaped confinement. This, however, is only observed when the number of particles $N$ ( 16 in this case) is commensurate to a square symmetry. For the case of 17 particles a couple of different structures are found. In Fig. 4b the additional particle is simply added at the bottom edge, while in Fig. 4c it is inserted at an interstitial site, hence considerably

\footnotetext{
'The mean square displacement in a two dimensional crysial diverges logarithmically wirh system sizc. Howevcr, for the sizes of systems considered in this work this divergence can be neglected.
} 


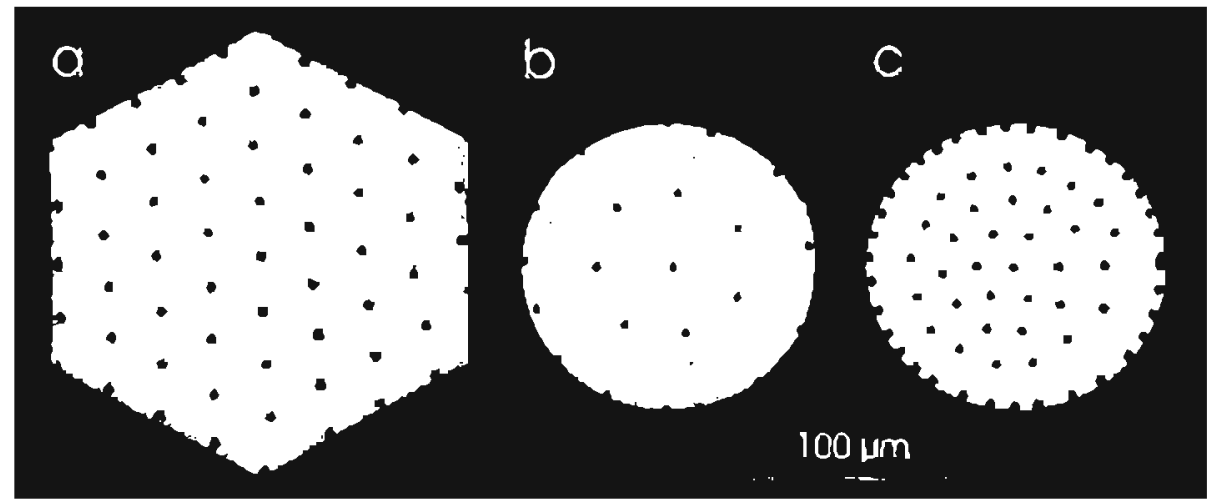

Fig. 3 Expcrimentally obscrved ground states for different geometries. (a) hexagonal ordering in a hexagonal confinement region. (b) Formation of one inner shell in a circular system of 21 particles. (c) Formation of three inner shells in a system of 71 particles io a circular confinement. On the right side. ihree paricles chains which arc marked aith arrows locally distort the structure. Thexe parlicle chains are formed by two or more coagulated particles and are alligned parallel to the direction of $B$ when the field is turned on. Because a chain has a larger magnetic moment $M$ than a single parricle it slightly distorts the lattice

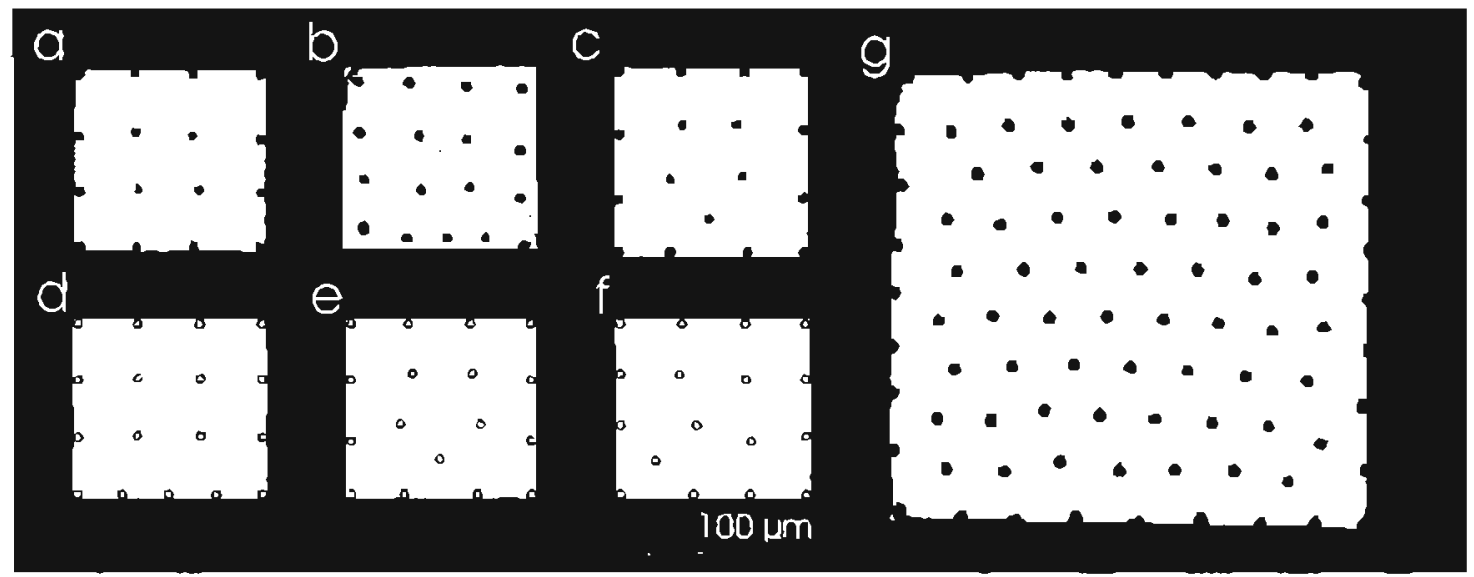

Fig. 4 Experimental results lor a square confinement of $90 \mu$ m sidelengh: (a) 16 parcicles ariange in a squarc $4 \times 4$ lallice. In (b) and (c) different arrangements for a system with $17=16+1$ particles are shown: (b) the additional particle moved to the bottom edge of the boundary, whereas in (c) a disiorted square lattice of 16 particles with an occupation of a subsquare is formed. The system (b) is slightly distorted due to imperfections of the PMMA film at the edges of the boundary. (d)-(g) Resulis for 17 particles achieved by simulated annealing

distorting its environment. In order to find out whether this states are degenerated or not we calculated their corresponding energies and additionally performed a simulated annealing computer calculation to derive the ground states. The results are shown in Figs. $4 d-f$. While Fig. 4d and e correspond to the experimental resulis of Fig. $4 b$ and $c$, we found no experimental match for $f$. The energies of Fig. $4 d-f$ are given as 1216,1223 and 1222 in arbitrary units, respectively. So Fig. $4 \mathrm{~d}$ is the ground state and Fig. 4e and ( are metastable states with an energy being about $0.5 \%$ larger than that of the ground state. This small difference might explain that we experimentally observe more than one structure (Fig. $4 \mathrm{~b}$ and c).

For larger particle numbers the influence of the boundary condition gets weaker. In Fig. $4 \mathrm{~g}$ we have plotted a picture of a square containing 98 particles. Here the system does not adapt a square lallice but has hexagonal structure with one lattice line aligned to the boundary. It is aoticeable that for this system size the hexagonal lattice is superior even with the wall-induced distortions visible on the left and right boundary of Fig. $4 \mathrm{~g}$.

\section{Circular symmetry}

As a last example we show systems with circular confinement regions, where, unlike as in the systems with hexagonal or square boundaries, the rotational symmetry of the confinement region is larger than the intrinsic symmetry of the lattice. Consequently, in this case the 


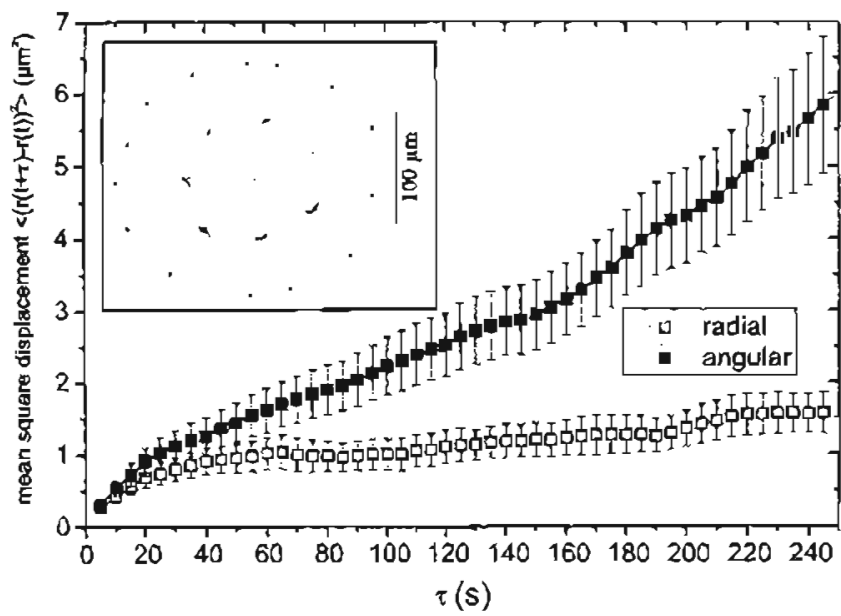

Fig. 5 Radial and angular components of the mean square displacements calculated by trajectories of 22 followed over 100 pictures in an interval of $5 \mathrm{~s}$. The trajoctories of the particles are shown in the inset. During the measurement two of the inner particles stuck to the substrate. This two particies and tbose which are in contact with the boundary (e) have not been included into the calculation

problems of commensurability are much smaller. Fig. $3 \mathrm{~b}$ and $\mathrm{c}$ show two systems with circular confinement and 21 and 71 particles, respectively. As can be seen the particles arrange in circular shells centered within the confinement region. This is consistent with computer simulations for 2D systems with both dipole-dipole [8] and Coulomb interactions [9] where the systems consist of shells which are filled consecutively with increasing particle number $N$, in analogy to a classical periodic system of elements.

Finally, after the discussion of static properties we want to present some first results about dynamics: In the inset of Fig. 5 we plotted the center-of-mass positions of the particles taken from 100 different video frames taken in an interval of $5 \mathrm{~s}$. From these trajectories we derived the angular and radial components of the mean squarc displacement and plotted the results in Fig. 5. It can be seen that the confinement results in a strongly anisotropic diffusion indicating that the mobility of the particles on the shells is by a factor of two larger than in the radial direction.

In conclusion, we performed experiments on the structure of $2 \mathrm{D}$ colloidal crystals in the presence of additional lateral confinement. It has been shown that the boundary conditions may completely dominate the structure and dynamics of small crystals.

The rather good agreement of our experimental results with computer simulations shows the reliability of the used superparamagnetic colloidal system as a model system for investigations of finite size effects in 2L 3 . An interesting topic for future reasearch is the freezing behaviour of small confined 2D crystals because in this case the sequence in which the system aquires bond orientational and cranslational order might be reversed.

Wo gratefully acknowledge financial support by the Deutsche Forschungsgemeinscbaft (SFB 513) and valuable discussions with K. Zahn and $G$. Maret.

\section{References}

1. Kosterlitz JM. Thouless DJ (1973) J Phys C 8:1181

2. Nelson DR, Halperin BI (1979) Phys Rev B $19: 2457$

3. Strandburg KJ (1988) Rev Mod Phys $60: 161$
4. Zahn K (1997) Phys Rev Lett 79:175

5. Faucheux LP, Libchaber AJ (1994) Pbys Rev E 49:5158

6. Bonsall L, Maradudin AA (1977) Phys Rev B 15:1959
7. Lubachevsky BD, Graham RL (1997) Discrete Comput Geom 18:179

8. Cebers A, Zhuk V (1987) Fizikas un teh. nisko zinātqu sērija $1: 112$

9. Bedanow VM, Peeters FM (1994) Phys Rev B 49:2667 